\title{
Development of a New Method for Assessing Global Risk of Alzheimer's Disease for Use in Population Health Approaches to Prevention
}

\author{
Kaarin J. Anstey • Nicolas Cherbuin • \\ Pushpani M Herath \\ Published online: 16 January 2013 \\ (C) The Author(s) 2013. This article is published with open access at Springerlink.com
}

\begin{abstract}
Alzheimer's disease (AD) affects approximately 35 million people worldwide. Increasing evidence suggests that many risk factors for $\mathrm{AD}$ are modifiable. AD pathology develops over decades. Hence risk reduction interventions require very long follow-ups to show effects on $\mathrm{AD}$ incidence. Focussing on AD risk, instead of diagnosis, provides a more realistic target for prevention strategies. We developed a novel methodology that yields a global approach to risk assessment for $\mathrm{AD}$ for use in population-based settings and interventions. The methodology was used to develop a risk assessment tool that can be updated as more evidence becomes available. First, a systematic search strategy identified risk and protective factors for AD. Eleven risk factors and four protective factors for $\mathrm{AD}$ were identified for which odds ratios were published or could be calculated (age, sex, education, body mass index, diabetes, depression, serum cholesterol, traumatic brain injury, smoking, alcohol intake, social engagement, physical activity, cognitive activity, fish intake, and pesticide exposure). An algorithm was developed to combine the odds ratios into an $\mathrm{AD}$ risk score. The approach allows for interactions among risk factors which provides for their varying impact over the life-course as current evidence suggests midlife is a critical period for some risk factors. Finally, a questionnaire was developed to assess the risk and protective factors by self-report. Compared with developing risk indices on single cohort studies, this approach allows for more risk factors to be included,
\end{abstract}

Electronic supplementary material The online version of this article (doi:10.1007/s11121-012-0313-2) contains supplementary material, which is available to authorized users.

K. J. Anstey $(\bowtie) \cdot$ N. Cherbuin $\cdot$ P. M. Herath

Centre for Research on Ageing, Health and Wellbeing, Bldg 63,

The Australian National University,

Canberra, ACT 0200, Australia

e-mail: kaarin.anstey@anu.edu.au greater generalizeability of results, and incorporation of interactions based on findings from different stages of the lifecourse.

Keywords Risk assessment · Alzheimer's disease · Systematic review $\cdot$ Risk index

Given the long prodromal period for Alzheimer's disease (AD), the lack of effective treatments, and the increasing prevalence associated with population aging, there is a need to identify population health approaches to reducing risk of the disease (Barnes et al. 2010). Information on risk factors for Alzheimer's disease is obtained from individual cohort studies and meta-analyses of these studies. These studies differ in terms of the number of exposures measured at baseline, the length of follow-up and the sample characteristics. Importantly, risk factors for AD may vary over the adult life-course. In studies comprising only older adults, there is potential confounding of the effects of preclinical $\mathrm{AD}$ on risk scores. For example, body weight declines up to 6 years prior to AD diagnosis (Anstey et al. 2011), as does serum cholesterol (Presecki et al. 2011). Cognitive decline and brain changes occur many years prior to diagnosis yet may be due to the disease and hence not independent risk factors. Hence, no single study provides all the information required for the development of an ideal risk assessment tool for Alzheimer's disease.

The aim of this study was to use an Evidence-Based Medicine approach to develop a new approach to risk assessment for Alzheimer's disease that incorporates all the currently reliable information on risk factors. This methodology differs from that previously used to develop risk assessment indices for dementia because it is based on review of the extant literature, rather than analysis of a 
single cohort study. We chose to restrict the tool to risk factors that could be assessed via self-report so that the index can be implemented online in primary care and intervention studies, and used in large-scale population health programs. The methodology could potentially be used in relation to other areas of chronic disease for which there is a wide literature and need for long-term population-based prevention strategies.

\section{Previous Risk Assessment Indices for Alzheimer's Disease and Dementia}

Two risk indices have been developed for AD specifically (as opposed for the more general category 'dementia'). These have each been based on follow-up of a sample of already older adults, to identify statistical associations between baseline measures and later diagnosis of AD. The first was developed by Reitz et al. (2010) using data from the Northern Manhattan study ( $n=1051$, mean age 75.66) and includes sex, education, ethnicity, apolipoprotein E genotype, diabetes, hypertension, smoking, high density lipoproteins, and waist to hip ratio (Reitz et al. 2010). Despite its strengths, this measure is not suitable for large-scale population-based studies because it depends on clinical assessment and genotyping. A second was developed from a German Primary Care patient registry and involved following up 3055 patients three times, at 18-month intervals. This study identified age, subjective memory complaint, cognitive function, depressive symptoms and Instrumental Activities of Daily Living (IADL) as predictors of conversion to AD (Jessen et al. 2011). Ideally, a risk assessment tool will not include screening measures or other indices of current mild cognitive impairment (subjective memory complaints, cognitive impairment, impairment in IADL) to allow for separation of risk factors from the predicted outcome. Mild cognitive impairment is often pre-clinical AD (Lopez et al. 2007). As cognitive decline is a core feature of dementia and $\mathrm{AD}$, inclusion of cognitive function, particularly indexed by a dementia screening instrument, would mean the risk index is for use after cognitive decline has commenced and hence is less predictive prior to the onset of symptoms. The optimal risk assessment tool for prevention trials will not include items that may also be symptomatic of the disease.

Two risk indices for the broader diagnosis of dementia (of which $\mathrm{AD}$ is the most common cause) have also been reported in the literature. Kivipelto et al. developed a dementia risk index using data from CAIDE study $(n=1449)$ in 2006. They found that age, sex, education, hypertension, high cholesterol and obesity predicted dementia risk over a 20 -year follow-up period (Kivipelto et al. 2006). The particular strength of this index was the validation over a long follow-up period and inclusion of middle-aged participants in the baseline assessment; however, it was restricted to the variables available in the baseline assessment of a single study. A second dementia risk index was calculated using data from the Cardiovascular Health Cognition Study $(n=$ 3608 , mean baseline age 76 years). In this study, age, poor cognitive performance, low BMI, APOE genotype, white matter hyperintensities on brain MRI, ventricular enlargement, thickening of the carotid artery, history of bypass surgery, slow physical performance, and abstaining from alcohol were risk factors for dementia over 6 years (Barnes et al. 2009). This scale is not suitable for population-based settings due to the inclusion of clinical measures. A brief version of this second risk index has also been reported (Barnes et al. 2010) and includes age, some simple cognitive tests, self-report of attentional difficulties, stroke, peripheral artery disease, coronary artery bypass surgery, low BMI, and lack of alcohol consumption. Although this shorter version requires less expensive clinical assessments, it still requires cognitive assessment and detailed medical history obtained from a health professional and is derived from a single cohort study of older adults.

Previous dementia and $\mathrm{AD}$ risk indices were limited to the variables included in the studies on which they were based and to the characteristics of their cohorts such as age at exposure (typically the age of the cohort at baseline assessment). Hence, they do not take into account the fact that some risk factors differ in their association with $A D$ over the adult life-course. For example, in mid-life both high cholesterol and high BMI are risk factors for AD but in latelife, neither is a risk factor for AD (Anstey et al. 2008, 2011). Moreover, some risk factors may also be affected by the disease itself. Weight loss typically precedes AD by about 6 years, and hence low BMI may show up as a risk factor for $\mathrm{AD}$ in a study of older adults (e.g., Barnes et al. 2009) when it is actually as symptom of AD (Knopman et al. 2007).

It is notable that the two previously published risk indices for dementia described above found that opposite categories of BMI were associated with increased risk of dementia. This is a concrete example of the limitations of basing an index on a single cohort, and the different findings resulting from different baseline ages of the cohorts. Using the broader data available from the literature on each risk factor allows for inclusion of interactions between age and specific risk factors to capture their differing effects over the adult life course.

The present study aimed to develop a methodology for creating a self-report Alzheimer's Disease Risk Index that could be updated as new findings become available. The overall AD risk assessment tool was called the Australian National University Alzheimer's Disease Risk Index (ANUADRI). There are three main advantages of this approach. 
First, it does not limit the possible number of risk factors that may be included in the index. Second, it derives from the principles of Evidence Based Medicine that assume the most reliable estimates of effect sizes are obtained from the statistical analysis of the population of studies (Davidoff et al. 1995; Lyman and Kuderer 2005). Third, it allows for the full information about a putative risk or protective factor to be considered when designing the tool (e.g., the age of exposure relevant to the risk factor, gender effects, optimal measurement characteristics). This project builds on a series of systematic reviews of risk factors for dementia and Alzheimer's disease conducted by the authors (Anstey et al. 2007, 2008, 2009, 2011).

\section{Method}

There were four stages to the development of the ANU-ADRI outlined in Fig. 1.

Development of a List of Putative Risk Factors and Protective Factors for AD that Can Be Measured by Self-report

The first stage (Fig. 1) involved collating an initial list of possible risk factors for inclusion. This was collated from the comprehensive review of risk factors for Alzheimer's Disease and Cognitive Decline published (Williams et al. 2010) and by the authors who had a thorough general knowledge of the literature obtained when conducting previous systematic reviews (Anstey et al. 2007, 2008, 2009, 2011). A systematic search of review articles on risk factors for $\mathrm{AD}$, and review articles on risk factors for $\mathrm{AD}$ were identified using PubMed search terms [Dementia OR
Alzheimer's disease OR Cognitive impairment] AND [Genetics, Age, Gender, Marital status, Family history of dementia, Family history of AD, Body Mass Index, Weight loss, Weight gain, Obesity, Occupation, Primary occupation, Diabetes mellitis, Depression, Hyperlipidaemia, Hypercholesterolemia, Cardiovascular disease, Myocardial Infarction, Angina, Ischemic heart disease, Heart failure, Cardiac failure, Arrhythmia, Atrial fibrillation, Stroke, Cerebrovascular accident, Transient ischemic attack, Hypertension, High blood pressure, Anxiety, Smoking, Alcohol, Omega 3 intake, Fish consumption, Physical activity, Exercise, Social network, Social engagement, Social activities, Cognitive leisure activities, Cognitive training, Brain training, Diet, Mediterranean diet, Fruit and vegetable intake, Total caloric intake, Fat intake, Caffeine, Antioxidants, Statin, anti-hypertensives, Hypertension treatments, Hormone Replacement Therapy, Oestrogen, Progesterone, NSAIDS, Vitamins, Nutritional supplements].

The second stage involve selecting risk and protective factors from the initial list for which there was reliable evidence of an association with AD. Inclusion criteria were that there was evidence for the risk factor derived from a high quality meta-analysis or multiple high quality cohort studies from which a pooled effect size could be calculated. High quality studies and meta-analyses were defined using the criteria applied in previous systematic reviews by the authors (Anstey et al. 2007, 2008, 2009, 2011; Williams et al. 2010). Briefly, they had to be prospective, have greater than 100 subjects, more than 12 months follow-up, include population-based samples and widely accepted research criteria used to diagnose AD (e.g., DSM-IV criteria). Factors for which high quality meta-analyses were available that showed no significant association between the putative risk

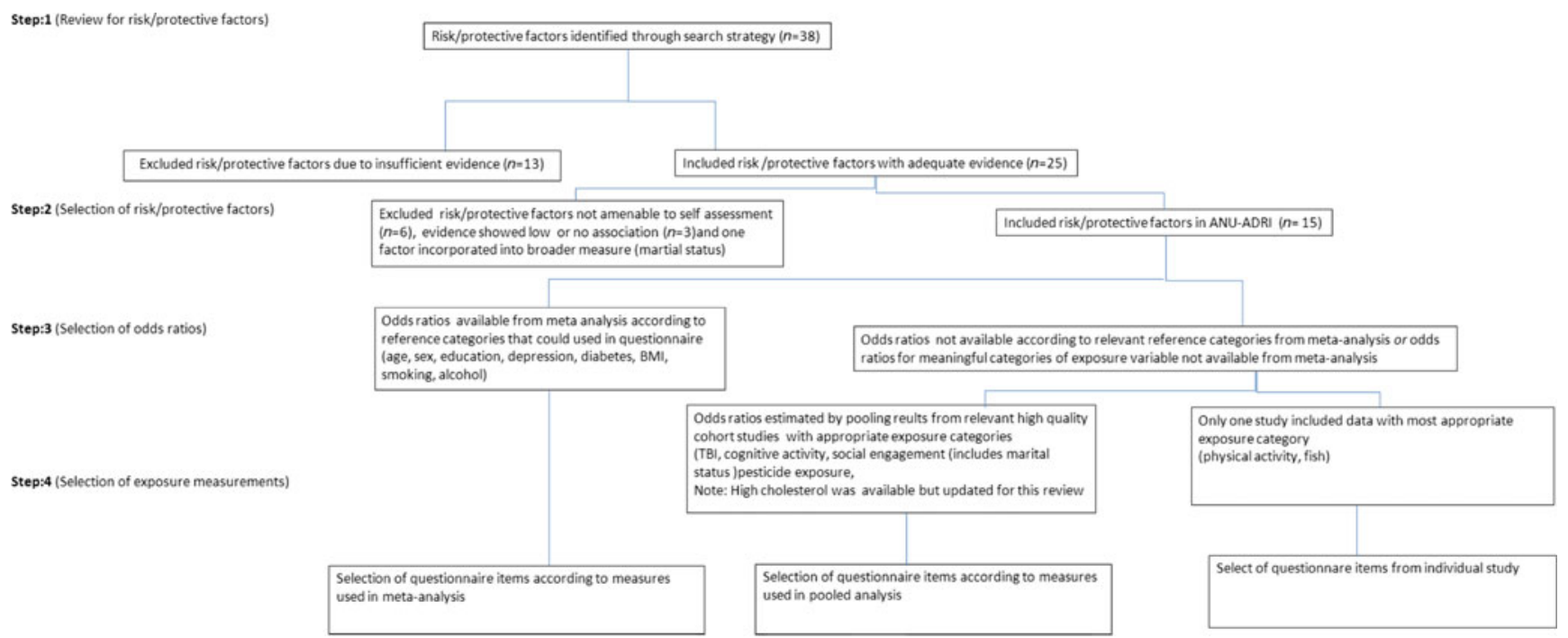

Fig. 1 Flowchart showing stages of development of the ANU-ADRI 
factor and $\mathrm{AD}$ were excluded (e.g., hypertension, nonsteroidal anti-inflammatory drugs).

Selection of Odds Ratios for Inclusion in the AD Risk Index

In the third stage, the odds ratios associated with risk and protective factors were derived from the literature using the following hierarchy of methods. First, odds ratios were extracted from systematic reviews where the exposure was clearly defined. If odds ratios were not available from systematic reviews, the authors identified high quality cohort studies and calculated odds ratios from pooled effect sizes. This occurred for education, traumatic brain injury, pesticide exposure, cognitive activity and social engagement (available online). If odds ratios were available but the exposure variable was not clearly defined in a manner that could be translated into a self-report questionnaire, the original and new high quality articles were located by authors and new pooled estimates calculated by the authors (e.g., high cholesterol and depressive symptoms) from articles reporting exposures that were translatable. In the case of physical activity, only one article contributing to the published pooled estimate reported odds ratios that could be translated into a measure and the results from this single article were used. In the case of cognitive activity, we obtained the raw data from authors and analysed it to determine cut-offs that could meaningfully be linked to cutoffs on the questionnaire used in these articles and then incorporated them into a self-report questionnaire. Where possible data were stratified by age of exposure, age of $\mathrm{AD}$ diagnosis, and sex.

\section{Classification of Protective Factors}

The ANU-ADRI includes both risk factors and protective factors rather than rescoring all factors as 'risk factors' because this reflects the way factors have been portrayed in the literature. Higher levels of physical activity, cognitive activity and high intake of fish are included as protective factors while low level of social engagement is included as a risk factor, consistent with the studies where these original findings were reported (Fotuhi et al. 2009; Fratiglioni et al. 2000; Williams et al. 2010; Wilson et al. 2007).

Selection of Questions for Inclusion in the AD Risk Assessment Tool

In the fourth stage, definitions of exposure variables for inclusion in the ANU Alzheimer's Disease Risk Index were determined. This led to the actual selection of questionnaire items for inclusion in the assessment tool (available online). Of risk factors identified for inclusion, the definitions of exposure variables were identified from the descriptions reported in articles reporting results of the relevant metaanalyses in the first instance. However, for some risk factors, the descriptions of the measures of the risk factors were not reported, but rather, generic categories created in the process of pooling the data were used in analyses (e.g., low physical activity versus high physical activity). In these instances we searched individual articles included in metaanalyses for exposure categories that could be linked to actual questionnaire items.

\section{Methodology for the ANU-ADRI Scoring System}

Individual ANU-ADRI scores were developed by creating an algorithm that sums the points attributed to individual risk factors using a previously published method (Kivipelto et al. 2006; Zammit et al. 2010). The points for each risk factor were obtained from the standardized beta-weights derived (from odds ratios of pooled effect sizes from metaanalyses). The lowest beta co-efficient $(0.13)$ was given a score of one and other beta weights were standardized by multiplying them by $1 / 0.13=7.6923$. An algorithm was written that sums the points attributed to individual risk factors (Kivipelto et al. 2006; Zammit et al. 2010) and incorporates interactions with age for relevant risk factors (high serum cholesterol, BMI) (Anstey et al. 2008, 2011).

\section{Results}

Thirty-eight potential risk factors were identified, of which 13 were excluded due to insufficient evidence. Of the 25 remaining risk factors, 6 were excluded because they could not be assessed via self-report, and one (marital status) was incorporated into a broader category of social engagement in the actual measurement instruments. Another three risk factors were eliminated due to lack of evidence of an association with $\mathrm{AD}$. This left 11 risk factors and 4 protective factors for inclusion in the ADRI. Table 1 reports the actual points attributed to each risk factor in the ANU-ADRI. Description of risk and protective factors and the source of effect sizes used in the ANU-ADRI are described below.

Demographics Odds ratios for age and gender were derived from population prevalence estimates of Alzheimer's disease in Australia, Europe and the USA (Anstey et al. 2010; Jorm et al. 2005; Access Economics 2009; Lobo et al. 2000; Ritchie and Kildea 1995). A systematic review of 16 cohort studies and 6 case control studies found a reliable association between low education and risk of $\mathrm{AD}(n=28025)$ (Caamano-Isorna et al. 2006). However, this review compared the lowest education group with the highest education group, and the highest education group with any other level 
Table 1 Characteristics and sources of variables included in the ANU-ADRI

\begin{tabular}{|c|c|c|c|}
\hline Characteristics & Beta weight & Points & Source of $\beta$ weight \\
\hline \multicolumn{4}{|l|}{ Age for males } \\
\hline$<65$ years & Reference & 0 & Anstey et al. 2010 \\
\hline 65 years- 70 & 0.13 & 1 & Jorm et al. 2005 \\
\hline 70 years- 75 & 1.57 & 12 & Access Economics 2009 \\
\hline 75 years- 80 & 2.04 & 18 & Lobo et al. 2000 \\
\hline 80 years- 85 & 3.37 & 26 & Ritchie and Kildea 1995 \\
\hline 85 years- 90 & 4.24 & 33 & \\
\hline$>90$ & 4.93 & 38 & \\
\hline \multicolumn{4}{|l|}{ Age for females } \\
\hline$<65$ years & Reference & 0 & \\
\hline 65 years- 70 & 0.64 & 5 & \\
\hline 70 years- 75 & 1.87 & 14 & \\
\hline 75 years- 80 & 2.75 & 21 & \\
\hline 80 years- 85 & 3.71 & 29 & \\
\hline 85 years- 90 & 4.58 & 35 & \\
\hline$>90$ years & 5.28 & 41 & \\
\hline \multicolumn{4}{|l|}{ Education } \\
\hline$>11$ years & Reference & 0 & Launer et al. 1999. Karp et al. 2004 \\
\hline 8 to 11 years & 0.42 & 3 & \\
\hline$<8$ years & 0.80 & 6 & \\
\hline \multicolumn{4}{|l|}{ BMI (age <60) } \\
\hline Normal & Reference & 0 & Anstey et al. 2011 \\
\hline Overweight & 0.30 & 2 & \\
\hline Obese & 0.71 & 5 & \\
\hline \multicolumn{4}{|l|}{ Diabetes } \\
\hline No diabetes & Reference & 0 & Lu et al. 2009 \\
\hline Diabetes & 0.33 & 3 & \\
\hline \multicolumn{4}{|l|}{ Symptoms of depression } \\
\hline CES-D $<16$ & Reference & 0 & Lenoir et al. 2004; Saczynski et al. 2010; Gatz et al. 2005; \\
\hline CES-D $>16$ Depression & 0.29 & 2 & Geerling et al. 2008; Dal Forno et al. 2005 \\
\hline \multicolumn{4}{|l|}{ High chol $($ aged $<60)$} \\
\hline Not high & Reference & 0 & Anstey et al. 2008; Mielke et al. 2010; Solomon et al. 2009 \\
\hline High & 0.41 & 3 & \\
\hline \multicolumn{4}{|l|}{ TBI } \\
\hline No TBI & Reference & 0 & Launer, et al. 1999; Plassman et al. 2000; Schofield et al. 1997 \\
\hline TBI & 0.46 & 4 & \\
\hline \multicolumn{4}{|l|}{ Smoking } \\
\hline Never & Reference & 0 & Anstey et al. 2007 \\
\hline Ever smoking & 0.19 & 1 & \\
\hline Current & 0.58 & 4 & \\
\hline \multicolumn{4}{|l|}{ Alcohol intake } \\
\hline No alcohol & Reference & 0 & Anstey et al. 2009 \\
\hline Light-mod & -0.33 & -3 & \\
\hline \multicolumn{4}{|l|}{ Soc. engagement } \\
\hline Highest & Reference & 0 & Fratiglioni et al. 2000; Saczynski et al. 2006 \\
\hline Lowest & 0.84 & 6 & \\
\hline Low to med & 0.51 & 4 & \\
\hline Med to high & 0.17 & 1 & \\
\hline
\end{tabular}


Table 1 (continued)

\begin{tabular}{|c|c|c|c|}
\hline Characteristics & Beta weight & Points & Source of $\beta$ weight \\
\hline \multicolumn{4}{|l|}{ Physical activity } \\
\hline Lowest & Reference & 0 & \multirow[t]{3}{*}{ Scarmeas et al. 2009} \\
\hline Medium & -0.29 & -2 & \\
\hline Higher level & -0.40 & -3 & \\
\hline \multicolumn{4}{|l|}{ Cognitive activity } \\
\hline Lowest & Reference & 0 & \multirow[t]{3}{*}{ Akbaraly et al. 2009; Wilson et al. 2007} \\
\hline Middle & -0.97 & -7 & \\
\hline Highest & -0.84 & -6 & \\
\hline \multicolumn{4}{|l|}{ Fish intake } \\
\hline$<0.25 \mathrm{p} \mathrm{p} /$ week & Reference & 0 & \multirow[t]{4}{*}{ Huang et al. 2005} \\
\hline $0.25-2 \mathrm{p} \mathrm{p} /$ week & -0.33 & -3 & \\
\hline $2-4 \mathrm{p} \mathrm{p} /$ week & -0.53 & -4 & \\
\hline$>4 \mathrm{p} \mathrm{p} /$ week & -0.62 & -5 & \\
\hline \multicolumn{4}{|l|}{ Pesticide exposure } \\
\hline Never & Reference & 0 & \multirow[t]{2}{*}{ Baldi et al. 2003; Hayden et al. 2010; Tyas et al. 2001} \\
\hline Ever & 0.31 & 2 & \\
\hline
\end{tabular}

The points were derived by multiply the beta weights by 7.6923 and rounding up to an integer; $\mathrm{TBI}=$ traumatic brain injury; soc. $=$ social; $\mathrm{p} p / \mathrm{week}=$ portions per week

of education; hence, the results did not relate to categories that were appropriate for the ANU-ADRI. We therefore pooled data from four European studies reported in the Eurodem analyses (Launer et al. 1999) and an additional study from a more recent meta-analysis (Caamano-Isorna et al. 2006; Karp et al. 2004) to produce the pooled estimate for education. Marital status was included as part of a social network measure described below and hence is not included as a separate demographic variable.

Diabetes Diabetes was identified as a risk factor for $\mathrm{AD}$ in a previous review (Williams et al. 2010). The odds ratio for diabetes was obtained from a systematic review of 8 studies $(n=23257)$ that identified diabetes mellitus by self report plus use of anti-diabetic medications, or laboratory tests (Lu et al. 2009; Williams et al. 2010).

Depression A meta-analysis of depression and AD $(n=$ 102172) (Ownby et al. 2006) found that depression is a risk factor for $\mathrm{AD}$. In this meta-analysis depression was defined according to diagnosis of depression or current symptoms and measures of depression included a range of clinical methods and scales, with validated cut-offs. In order to identify an odds ratio for use in the ANU-ADRI, we investigated both history of depression, and depression measured by symptom scales as a risk factor for $\mathrm{AD}$ because these were both obtained by self-report. We re-analysed the data from this meta-analysis and our review to pool four studies reporting a history of depression (Gatz et al. 2005; Geerlings et al. 2008; Li et al. 2001; Lindsay et al. 2002) and found that self-reported history of depression was not associated with AD. Of all symptom scales that could be administered by self-report, the Centre for Epidemiological Studies Depression Scale (CES-D 20-item scale) was included in the greatest number of cohort studies reporting effect sizes for the association between depressive symptoms and $\mathrm{AD}$ reflecting the wide use of this measure in high quality epidemiological studies. We therefore selected all studies reporting associations between depression and $\mathrm{AD}$ where depression had been measured using the CES-D from the Ownby et al. meta-analysis, and also searched the literature and identified two relevant studies that had been published after this review was published. The effect size for depression as a risk factor for $\mathrm{AD}$ was computed from five studies (Dal Forno et al. 2005; Gatz et al. 2005; Geerlings et al. 2008; Li et al. 2001; Lindsay et al. 2002) and the 20-item CES-D was included in the ANU-ADRI.

Overweight BMI and Obesity Odds ratios for mid-life overweight BMI and obese BMI were obtained from our recent meta-analysis of 15 cohort studies including 25624 participants followed for AD. BMI categories were classified using the WHO criteria (Sabia et al. 2009).

Brain Injury Our search of the literature identified traumatic brain injury (TBI) as a potential risk factor for AD. However, the only published meta-analysis reported a pooled estimates for 15 case control studies including 4525 participants (Fleminger et al. 2003). To obtain an estimate based on prospective cohort studies, we identified three publications 
reporting effect sizes for $\mathrm{TBI}$ as a risk factor for $\mathrm{AD}$ and estimated a pooled effect size from these (Launer et al. 1999; Plassman et al. 2000; Schofield et al. 1997). The forest plot for this analyses is included online.

Smoking Odds ratios for current versus never smokers, and current versus former smokers were obtained from our previous meta-analysis of 19 prospective studies including 13786 participants followed for AD (Anstey et al. 2007).

Cholesterol Total serum cholesterol level in mid-life is recognized as a risk factor for AD (Anstey et al. 2008); however, studies vary in how the exposure variable is measured and whether they use self-report or serum measures of high cholesterol. For this study we updated our previously published analyses and derived an odds ratio for self-reported high cholesterol as a risk factor for AD based on the published data for serum levels of cholesterol (National Institutes of Health 2002). We chose a cut-off of $<6.2$ (or less than $240 \mathrm{mg} / \mathrm{dl}$ ) for "normal" and $>6.2$ for high total cholesterol based on recommendations from the Adult Treatment Panel (ATP) III guidelines (National Institutes of Health 2002) (Solomon et al. 2009). We estimated the odds ratio from a meta-analysis that included the results from articles included in our previously published systematic review (Williams et al. 2010) as well as recently published articles (Mielke et al. 2010; Solomon et al. 2009) and involved follow-up data on 15780 participants (from 5 studies). The forest plot for this analysis is shown in the online supplement.

Physical Activity There is consistent evidence that physical activity reduces the risk of AD from meta-analyses (Hamer and Chida 2009; Williams et al. 2010). However, no metaanalysis of physical activity as a risk factor for AD reported information on the exposure variables in a way that could be translated into an available self-report measure of physical activity. Hamer and Chida (2009) identified only five studies reporting an association between physical activity and AD and only one of these incorporated both intensity and frequency of physical activity (Laurin et al. 2001). This study used questions that had not been validated and were unsuitable for inclusion in the ANU-ADRI.

On the basis that the literature does support the inclusion of physical activity as a protective factor in the ANU-ADRI, we decided to search for odds ratios from studies that reported their findings in relation to specific measures of physical activity that could be incorporated into a questionnaire. Given the aim of the ANU-ADRI for use in clinical trials and to guide advice on risk reduction, we aimed to include three levels of physical activity in the questionnaire, and hence odds ratios needed to be available for medium and high levels of physical activity compared to none. Only one study was identified that reported odds ratios for levels of activity that included a full range of categories and could be translated into cut-offs on a questionnaire (Scarmeas et al. 2009). Hence these odds ratios were selected. The Scarmeas et al. (2009) study used the Gordin Leisure time exercise questionnaire and this measure has shown a strong association with the International Physical Activity Questionnaire (IPAQ) (Sandroff et al. 2012; Weikert et al. 2012). Scarmeas et al. defined three levels of physical activity using Metabolic Equivalent (MET). For the ANU-ADRI we chose the IPAQ as a measure of physical activity because it includes a more detailed assessment than the Gordin scale, and unlike the Gordin scale has been validated in 12 countries ensuring greater generalizeability and validity of the items (Craig et al. 2003). The ANU-ADRI point system defines three levels of physical activity using MET values according to the IPAQ scoring guidelines.

Alcohol Consumption Odds ratios for alcohol consumption were obtained from our meta-analysis of 15 studies including 14646 participants (Anstey et al. 2009). This review showed that alcohol abstainers had an increased risk of AD compared to light to moderate drinkers. In this review, reference categories were based on those used in our metaanalysis which corresponded to the current Australian National Health and Medical Research Council categories. In the ANU-ADRI, the AUDIT questionnaire is used to assess the number of drinks per week and this instrument provides the information required to generate the alcohol consumption categories associated with the odds ratios reported in our review.

Fish Consumption The only dietary factor reliably linked to the risk of $\mathrm{AD}$ was fish consumption. This was found to be protective against cognitive decline and AD (BarbergerGateau et al. 2007; Huang et al. 2005; Williams et al. 2010). A recent review of five cohort studies reported that four showed a protective effect of fish intake or long-chain omega-3 fatty acid supplements on AD. It was not possible to pool these estimates of the effect of fish intake on $\mathrm{AD}$ because of differences between studies in the measure of exposure to fish, and the reporting of results. For the purpose of the ANU-ADRI, individual study questionnaires were reviewed to identify the most suitable self-report questions that assess fish intake that had been validated, and was freely available. Based on this review the questions and odds ratios for fish consumption were selected from the Cardiovascular Health Study (Huang et al. 2005).

Cognitive Activity Cognitive activity was identified by Williams et al. (2010) as a factor that reduced the risk of 
AD. Two large cohort studies reported a reduced risk of $\mathrm{AD}$ in older adults who engage in cognitively stimulating activities (Akbaraly et al. 2009; Wilson et al. 2007). The odds ratio for cognitive activity was calculated from these two studies, with data obtained from authors for one study (Wilson et al. 2007). (Forest plot included in online supplement). Both studies used similar methodology to evaluate exposure level. The questionnaire developed for the Rush Memory and Aging Project has been used in three studies by the same research group and has adequate short-term temporal stability and internal consistency. It was therefore chosen for inclusion in the ANU-ADRI; however, items were adapted to include online cognitive activities (e.g., accessing libraries online in addition to in person).

Social Engagement Social engagement was identified by the Williams report as protective against Alzheimer's disease with results available from four large cohort studies reporting effect sizes for social engagement (Bassuk et al. 1999; Fratiglioni et al. 2000; Helmer et al. 1999; Saczynski et al. 2006). Two of these used similar measures (HAAS and Kungsholmen) and data from these were pooled (see online supplement) to obtain the odds ratios for the ANU-ADRI (Fratiglioni et al. 2000; Saczynski et al. 2006). The social network measure included marital status, size and quality of social network, level of social activities and living arrangements for both studies and the same measure was included in the ANU-ADRI.

Occupational Exposure to Pesticide Occupational exposure to pesticide as a risk factor for $\mathrm{AD}$ was identified in the review by Williams et al. (2010). We identified an additional article reporting an odds ratio for occupational pesticide exposure (Baldi et al. 2003; Hayden et al. 2010; Tyas et al. 2001) and a pooled estimate was calculated based on these (included in the online supplement). Questions were developed specifically for the ANU-ADRI.

Variables Not Included Several studies reported that coronary artery disease was a risk factor for $\mathrm{AD}$; however, this factor was measured using incompatible methods and metaanalysis of findings was not possible. There is still not enough evidence to evaluate whether ischemic heart disease (including myocardial infarction, angina, and stroke) is a risk factor for $\mathrm{AD}$. With respect to stroke it is difficult to differentiate between $\mathrm{AD}$ and post-stroke vascular $\mathrm{AD}$ and we did not find any systematic review that could clarify this issue. There is still no firm evidence that hypertension is a risk factor for $\mathrm{AD}$ according to the most recent metaanalysis (Power et al. 2011). While ethnicity is included in a previous dementia risk index (Reitz et al. 2010), there is no meta-analysis of ethnicity and it is unknown whether this finding generalizes to other studies or the population. We identified no meta-analysis of self-reported family history of $\mathrm{AD}$ based on prospective studies, despite one metaanalysis of case control studies finding an association (van Duijn et al. 1991).

\section{Assumptions Made in the ANU-ADRI}

Due to the limitations of available evidence specific assumptions are made in the ANU-ADRI that will be evaluated in later versions as more data become available. Assumptions are made about the equivalence for most risk factors for males and females as few studies report odds ratios for males distinct from females. Some assumptions are made about the effect of some risk factors occurring at ages younger than that observed in the studies from which odds ratios were obtained. These assumptions were considered carefully based on available knowledge of the mechanisms by which risk factors may affect AD. For example, the systematic review of smoking included mostly studies of older adults so that the odds ratios reported in Anstey et al. (2007) apply to older adults but not middle-aged adults. However, no age limit is specified for smoking in the ANUADRI. This is because the ANU-ADRI is aimed to assess risk of $\mathrm{AD}$ on the assumption that a person's risk profile remained constant until old age. (i.e., if an individual who smokes in late-middle age does not give up smoking then their risk of $\mathrm{AD}$ in old age will increase by $79 \%$ ). Assumptions were also made in relation to the equivalence of clinical and self-report measures of high cholesterol in order to apply the risk ratio from studies using serum cholesterol to the self-report data on high cholesterol. The additive nature of the algorithm developed to compute the ANU-ADRI assumes that the interactions between risk factors are negligible. The analyses from individual cohort studies reporting associations between risk factors and $\mathrm{AD}$ adjust for covariates, which to some extent controls for possible covariation. This assumption of additivity of risk factors can be tested when validation studies of the ANU-ADRI are conducted.

\section{Comparison with Previous AD Risk Assessment Tools}

Reitz et al. (2010) focused on vascular risk factors for AD. Of the 11 risk factors included in their AD risk score, 6 could be ascertained by self-report including age, sex, education, ethnicity, diabetes, heart disease. The odds ratio for diabetes was higher in this study than that derived from meta-analyses and used in the ANU-ADR (2.04 vs 1.39). This study did not find smoking significantly associated with risk of $\mathrm{AD}$ but included ethnicity and heart disease which are not included in the ANU-ADRI. Jessen et al. found five variables predicted conversion to $\mathrm{AD}$, three of which could be assessed by self-report (age, memory 
complaint and difficulties with instrumental activities of daily living). The latter two could be caused by $\mathrm{AD}$ and hence are not necessarily independent risk factors.

\section{Discussion}

We report a new methodology for developing evidencebased risk assessment tools focussing on the risk assessment of AD. This method is comprehensive, and incorporates a life-course perspective into risk assessment by allowing for risk and protective factors to interact with age. We argue that this evidence-based approach to risk assessment for a chronic disease that is highly prevalent in late-life provides the best foundation for prevention programs aimed at reducing population-prevalence through targeted risk reduction. An evidence-based risk assessment incorporating age-specific effects may also be used to provide individual tailored advice by primary care physicians in counselling patients. We acknowledge that there is also a role for risk assessment tools incorporating clinical measures that are predictive of disease or indicative of pre-clinical dementia but this falls outside the aims of the current study.

Our approach identified far more risk and protective factors than previous studies that have developed risk indices based on individual cohort studies. This increases the utility of the instrument for use in population-based primary prevention programs. It may be used to screen individuals to identify those at high risk of $\mathrm{AD}$ who would benefit most from intervention programs. The tool may also be used as an outcome measure for randomized controlled trials of AD risk reduction. The methodology we have presented provides a framework for continual updating and improving of the risk assessment. As more studies are published, the odds ratios can be recalculated and the scoring algorithm updated. As new risk and protective factors are identified they can be added. This methodology could be applied in other settings where there is a need to develop population-level approaches to prevention and incorporate information on risk factors that varies over the life-course.

Biological mechanisms for most of the risk factors included in the ANU-ADRI have been identified or else biologically plausible pathways have been outlined and are described elsewhere. For example, the proposed pathophysiological mechanisms underlying the role of diabetes in the development of $\mathrm{AD}$ include endocrine, metabolic and vascular abnormalities and dysfunctions (Biessels et al. 2006). TBI is thought to increase the risk of AD by accelerating amyloid deposition (Kidd 2008). The protective effect of alcohol has been demonstrated in a mouse model and has been attributed to the attenuation of $\beta$ neuropathy following alcohol consumption (Wang et al. 2006). The mechanisms by which social and cognitive stimulation are thought to reduce the risk of $\mathrm{AD}$ remain unknown but are thought to involve neurogenesis resulting from neurostimulation (Redolat and Mesa-Gresa 2011), and down regulation of stress pathways (Rodriguez et al. 2011).

Our survey of the literature did not identify any study that includes all the exposure variables measured by the ANUADRI; hence, we expect that validation studies will underestimate the predictive validity of the index. This research however may be used to guide the design of ongoing and new cohort studies focusing in cognitive decline and dementia as outcomes. Estimation of predictive validity from existing datasets containing large numbers of risk and protective factors is the best method available to assess the utility of the index, and to derive valid ranges on the index that correspond to 'low risk,' 'moderate risk' and 'high risk'. External validation of the ANU-ADRI on three such cohort studies is in progress.

Acknowledgements This work was funded by the Australian Dementia Collaborative Research Centres. Anstey and Cherbuin are funded by National Health and Medical Research Council fellowships 1002560 and 471501 . There are no conflicts of interest. We thank the authors who kindly provided data for meta-analyses or re-analysis to obtain odds ratios.

Open Access This article is distributed under the terms of the Creative Commons Attribution License which permits any use, distribution, and reproduction in any medium, provided the original author(s) and the source are credited.

\section{References}

Access Economics (2009) Keeping Dementia Front of Mind: Incidence and Prevalence 2009. Report prepared for Alzheimer's Australia. Canberra: Access Economics Pty Limited.

Akbaraly, T. N., Portet, F., Fustinoni, S., Dartigues, J. F., Artero, S., Rouaud, O., \& Berr, C. (2009). Leisure activities and the risk of dementia in the elderly: Results from the Three-City Study. Neurology, 73, 854-861.

Anstey, K. J., von Sanden, C., Salim, A., \& O'Kearney, R. (2007). Smoking as a risk factor for dementia and cognitive decline: A meta-analysis of prospective studies. American Journal of Epidemiology, 166, 367-378.

Anstey, K. J., Lipnicki, D. M., \& Low, L. F. (2008). Cholesterol as a risk factor for dementia and cognitive decline: A systematic review of prospective studies with meta-analysis. The American Journal of Geriatric Psychiatry, 16, 343-354.

Anstey, K. J., Mack, H. A., \& Cherbuin, N. (2009). Alcohol consumption as a risk factor for dementia and cognitive decline: Meta-analysis of prospective studies. The American Journal of Geriatric Psychiatry, 17, 542-555. doi:10.1097/JGP.0b013e 3181a2fd07.

Anstey, K. J., Burns, R. A., Birrell, C. L., Steel, D., Kiely, K. M., \& Luszcz, M. A. (2010). Estimates of probable dementia prevalence from population-based surveys compared with dementia prevalence estimates based on meta-analyses. BMC Neurol, 10, 62.

Anstey, K. J., Cherbuin, N., Budge, M., \& Young, J. (2011). Body mass index in midlife and late-life as a risk factor for dementia: A meta- 
analysis of prospective studies. Obesity Reviews, 12, e426-37. doi:10.1111/j.1467-789X.2010.00825.x.

Baldi, I., Lebailly, P., Mohammed-Brahim, B., Letenneur, L., Dartigues, J. F., \& Brochard, P. (2003). Neurodegenerative diseases and exposure to pesticides in the elderly. American Journal of Epidemiology, 157, 409-414.

Barberger-Gateau, P., Raffaitin, C., Letenneur, L., Berr, C., Tzourio, C., Dartigues, J. F., \& Alperovitch, A. (2007). Dietary patterns and risk of dementia - The three-city cohort study. Neurology, 69, 1921-1930.

Barnes, D. E., Covinsky, K. E., Whitmer, R. A., Kuller, L. H., Lopez, O. L., \& Yaffe, K. (2009). Predicting risk of dementia in older adults: The late-life dementia risk index. Neurology, 73, 173-179.

Barnes, D. E., Covinsky, K. E., Whitmer, R. A., Kuller, L. H., Lopez, O. L., \& Yaffe, K. (2010). Commentary on "Developing a national strategy to prevent dementia: Leon Thal Symposium 2009." Dementia risk indices: A framework for identifying individuals with a high dementia risk. Alzheimers and Dementia, $6,138-141$

Bassuk, S. S., Glass, T. A., \& Berkman, L. F. (1999). Social disengagement and incident cognitive decline in community-dwelling elderly persons. Annals of Internal Medicine, 131, 165-173.

Biessels, G. J., Staekenborg, S., Brunner, E., Brayne, C., \& Scheltens, P. (2006). Risk of dementia in diabetes mellitus: A systematic review. Lancet Neurology, 5, 64-74.

Caamano-Isorna, F., Corral, M., Montes-Martinez, A., \& Takkouche, B. (2006). Education and dementia: A meta-analytic study. Neuroepidemiology, 26, 226-232.

Craig, C. L., Marshall, A. L., Sjostrom, M., Bauman, A. E., Booth, M. L., Ainsworth, B. E., Oja, P. (2003). International physical activity questionnaire: 12-country reliability and validity. Medical Science Sports Exercise, 35, 1381-1395.

Dal Forno, G., Palermo, M. T., Donohue, J. E., Karagiozis, H., Zonderman, A. B., \& Kawas, C. H. (2005). Depressive symptoms, sex, and risk for Alzheimer's disease. Annals of Neurology, 57, 381-387. doi:10.1002/ana.20405.

Davidoff, F., Haynes, B., Sackett, D., \& Smith, R. (1995). Evidence based medicine. BMJ, 310, 1085-1086.

Fleminger, S., Oliver, D. L., Lovestone, S., Rabe-Hesketh, S., \& Giora, A. (2003). Head injury as a risk factor for Alzheimer's disease: The evidence 10 years on; a partial replication. Journal of Neurology, Neurosurgery, and Psychiatry, 74, 857-862.

Fotuhi, M., Mohassel, P., \& Yaffe, K. (2009). Fish consumption, longchain omega-3 fatty acids and risk of cognitive decline or Alzheimer disease: A complex association. Nature Clinical Practice Neurology, 5, 140-152. doi:10.1038/ncpneuro1044.

Fratiglioni, L., Wang, H. X., Ericsson, K., Maytan, M., \& Winblad, B. (2000). Influence of social network on occurrence of dementia: A community-based longitudinal study. Lancet, 355, 1315-1319.

Gatz, J. L., Tyas, S. L., St John, P., \& Montgomery, P. (2005). Do depressive symptoms predict Alzheimer's disease and dementia? Journal of Gerontology Series A Biological Science and Medical Science, 60, 744-747.

Geerlings, M. I., den Heijer, T., Koudstaal, P. J., Hofman, A., \& Breteler, M. M. (2008). History of depression, depressive symptoms, and medial temporal lobe atrophy and the risk of Alzheimer disease. Neurology, 70, 1258-1264.

Hamer, M., \& Chida, Y. (2009). Physical activity and risk of neurodegenerative disease: A systematic review of prospective evidence. Psychological Medicine, 39, 3-11.

Hayden, K. M., Norton, M. C., Darcey, D., Ostbye, T., Zandi, P. P., Breitner, J. C., \& Welsh-Bohmer, K. A. (2010). Occupational exposure to pesticides increases the risk of incident AD: The Cache County study. Neurology, 74, 1524-1530.

Helmer, C., Damon, D., Letenneur, L., Fabrigoule, C., BarbergerGateau, P., Lafont, S., et al. (1999). Marital status and risk of
Alzheimer's disease: A French population-based cohort study. Neurology, 53, 1953-1958.

Huang, T. L., Zandi, P. P., Tucker, K. L., Fitzpatrick, A. L., Kuller, L. H., Fried, L. P., et al. (2005). Benefits of fatty fish on dementia risk are stronger for those without APOE epsilon4. Neurology, 65, $1409-1414$.

Jessen, F., Wiese, B., Bickel, H., Eifflander-Gorfer, S., Fuchs, A., Kaduszkiewicz, H., et al. (2011). Prediction of dementia in primary care patients. PLoS One, 6, e16852. doi:10.1371/journal. pone. 0016852 .

Jorm, A. F., Dear, K. B., \& Burgess, N. M. (2005). Projections of future numbers of dementia cases in Australia with and without prevention. [Comparative Study]. The Australian and New Zealand journal of psychiatry, 39, 959-963. doi:10.1111/j.1440-1614.2005.01713.x.

Karp, A., Kareholt, I., Qiu, C., Bellander, T., Winblad, B., \& Fratiglioni, L. (2004). Relation of education and occupation-based socioeconomic status to incident Alzheimer's disease. American Journal of Epidemiology, 159, 175-183.

Kidd, P. M. (2008). Alzheimer's Disease, amnestic mild cognitive impairment, and age-associated memory impairment: Current understanding and progress toward integrative prevention. Alternative Medicine Review, 13, 85-115.

Kivipelto, M., Ngandu, T., Laatikainen, T., Winblad, B., Soininen, H., \& Tuomilehto, J. (2006). Risk score for the prediction of dementia risk in 20 years among middle aged people: A longitudinal, population-based study. Lancet Neurology, 5, 735-741.

Knopman, D. S., Edland, S. D., Cha, R. H., Petersen, R. C., \& Rocca, W. A. (2007). Incident dementia in women is preceded by weight loss by at least a decade. Neurology, 69, 739-746. doi:10.1212/ 01.wnl.0000267661.65586.33.

Launer, L. J., Andersen, K., Dewey, M. E., Letenneur, L., Ott, A., Amaducci, L. A., et al. (1999). Rates and risk factors for dementia and Alzheimer's disease: Results from EURODEM pooled analyses. EURODEM Incidence Research Group and Work Groups. European Studies of Dementia. Neurology, 52, 78-84.

Laurin, D., Verreault, R., Lindsay, J., MacPherson, K., \& Rockwood, K. (2001). Physical activity and risk of cognitive impairment and dementia in elderly persons. Archives of Neurology, 58, 498-504.

Lenoir, H., Dufouil, C., Auriacombe, S., Lacombe, J. M., Dartigues, J. F., Ritchie, K., \& Tzourio, C. (2004). Depression history, depressive symptoms, and incident dementia: The 3C Study. Journal of Alzheimers Disease, 26, 27-38.

Li, Y. S., Meyer, J. S., \& Thornby, J. (2001). Longitudinal follow-up of depressive symptoms among normal versus cognitively impaired elderly. International Journal of Geriatric Psychiatry, 16, 718727.

Lindsay, J., Laurin, D., Verreault, R., Hebert, R., Helliwell, B., Hill, G. B., \& McDowell, I. (2002). Risk factors for Alzheimer's disease: A prospective analysis from the Canadian Study of Health and Aging. American Journal of Epidemiology, 156, 445-453.

Lobo, A., Launer, L. J., Fratiglioni, L., Andersen, K., Di Carlo, A., Breteler, M. M., Hofman, A. (2000). Prevalence of dementia and major subtypes in Europe: A collaborative study of populationbased cohorts. Neurologic Diseases in the Elderly Research Group. [Multicenter Study Research Support, Non-U.S. Gov't]. Neurology, 54, S4-9.

Lopez, O. L., Kuller, L. H., Becker, J. T., Dulberg, C., Sweet, R. A., Gach, H. M., \& Dekosky, S. T. (2007). Incidence of dementia in mild cognitive impairment in the Cardiovascular Health Cognition Study. Archives of Neurology, 64, 416-420.

Lu, F. P., Lin, K. P., \& Kuo, H. K. (2009). Diabetes and the risk of multi-system aging phenotypes: A systematic review and metaanalysis. PLoS One, 4. doi:10.1371/journal.pone.0004144.

Lyman, G. H., \& Kuderer, N. M. (2005). The strengths and limitations of meta-analyses based on aggregate data. BMC Medical Research Methodology, 5, 14. 
Mielke, M. M., Zandi, P. P., Shao, H., Waern, M., Ostling, S., Guo, X., \& Gustafson, D. R. (2010). The 32-year relationship between cholesterol and dementia from midlife to late life. Neurology, 75, 1888-1895. doi:10.1212/WNL.0b013e3181feb2bf.

National Institutes of Health (2002). National Cholesterol Education Program Expert Panel on Detection, Evaluation and Treatment of High Blood Cholesterol in Adults (Adult Treatment Panel III): Final Report. Bethesda, MD: National Institutes of Health.

Ownby, R. L., Crocco, E., Acevedo, A., John, V., \& Loewenstein, D. (2006). Depression and risk for Alzheimer disease - Systematic review, meta-analysis, and metaregression analysis. Archives of General Psychiatry, 63, 530-538.

Plassman, B. L., Havlik, R. J., Steffens, D. C., Helms, M. J., Newman, T. N., Drosdick, D., et al. (2000). Documented head injury in early adulthood and risk of Alzheimer's disease and other dementias. Neurology, 55, 1158-1166.

Power, M. C., Weuve, J., Gagne, J. J., McQueen, M. B., Viswanathan, A., \& Blacker, D. (2011). The association between blood pressure and incident Alzheimer disease: A systematic review and meta-analysis. Epidemiology, 22, 646-659. doi:10.1097/EDE.0b013e31822708b5.

Presecki, P., Muck-Seler, D., Mimica, N., Pivac, N., Mustapic, M., Stipcevic, T., \& Smalc, V. F. (2011). Serum lipid levels in patients with Alzheimer's disease. Collegium Antropologicum, 35(Suppl 1), 115-120.

Redolat, R., \& Mesa-Gresa, P. (2011). Potential benefits and limitations of enriched environments and cognitive activity on age-related behavioural decline. Current Topics in Behavioral Neuroscience. doi:10.1007/7854 2011134.

Reitz, C., Tang, M. X., Schupf, N., Manly, J. J., Mayeux, R., \& Luchsinger, J. A. (2010). A summary risk score for the prediction of Alzheimer disease in elderly persons. Archives of Neurology, $67,835-841$.

Ritchie, K., \& Kildea, D. (1995). Is senile dementia "age-related" or "ageing-related"? - evidence from meta-analysis of dementia prevalence in the oldest old. [Meta-Analysis]. Lancet, 346, 931-934.

Rodriguez, J. J., Noristani, H. N., Olabarria, M., Fletcher, J., Somerville, T. D., Yeh, C. Y., \& Verkhratsky, A. (2011). Voluntary running and environmental enrichment restores impaired hippocampal neurogenesis in a triple transgenic mouse model of Alzheimer's disease. Current Alzheimers Research, 8, 707-717.

Sabia, S., Kivimaki, M., Shipley, M. J., Marmot, M. G., \& SinghManoux, A. (2009). Body mass index over the adult life course and cognition in late midlife: The Whitehall II cohort study. American Journal of Clinical Nutrition, 89, 601-607. doi:10.3945/ajen.2008.26482.

Saczynski, J. S., Beiser, A., Seshadri, S., Auerbach, S., Wolf, P. A., \& $\mathrm{Au}$, R. (2010). Depressive symptoms and risk of dementia: The Framingham Heart Study. Neurology, 75, 35-41.
Saczynski, J. S., Pfeifer, L. A., Masaki, K., Korf, E. S., Laurin, D., White, L., \& Launer, L. J. (2006). The effect of social engagement on incident dementia: The Honolulu-Asia aging study. American Journal of Epidemiology, 163, 433-440.

Sandroff, B. M., Dlugonski, D., Weikert, M., Suh, Y., Balantrapu, S., \& Motl, R. W. (2012). Physical activity and multiple sclerosis: New insights regarding inactivity. Acta Neurologica Scandinavica, 126, 256-262. doi:10.1111/j.1600-0404.2011.01634.x

Scarmeas, N., Luchsinger, J. A., Schupf, N., Brickman, A. M., Cosentino, S., Tang, M. X., \& Stern, Y. (2009). Physical activity, diet, and risk of Alzheimer disease. JAMA, 302, 627-637.

Schofield, P. W., Tang, M., Marder, K., Bell, K., Dooneief, G., Chun, M., Mayeux, R., et al. (1997). Alzheimer's disease after remote head injury: An incidence study. Journal of Neurology, Neurosurgery, and Psychiatry, 62, 119-124.

Solomon, A., Kivipelto, M., Wolozin, B., Zhou, J., \& Whitmer, R. A. (2009). Midlife serum cholesterol and increased risk of Alzheimer's and vascular dementia three decades later. Dementia and Geriatric Cognitive Disorders, 28, 75-80. doi:10.1159/ 000231980

Tyas, S. L., Manfreda, J., Strain, L. A., \& Montgomery, P. R. (2001). Risk factors for Alzheimer's disease: A population-based, longitudinal study in Manitoba, Canada. International Journal of Epidemiology, 30, 590-597.

van Duijn, C. M., Clayton, D., Chandra, V., Fratiglioni, L., Graves, A. B., Heyman, A., et al. (1991). Familial aggregation of Alzheimer's disease and related disorders: A collaborative re-analysis of casecontrol studies. International Journal of Epidemiology, 20(Suppl 2), S13-S20.

Wang, J., Ho, L., Zhao, Z., Seror, I., Humala, N., Dickstein, D., et al. (2006). Moderate consumption of Cabernet Sauvignon attenuates Abeta neuropathology in a mouse model of Alzheimer's disease. The FASEB Journal, 20, 2313-2320.

Weikert, M., Suh, Y., Lane, A., Sandroff, B., Dlugonski, D., Fernhall, B., \& Motl, R. W. (2012). Accelerometry is associated with walking mobility, not physical activity, in persons with multiple sclerosis. Medical Engineering \& Physics, 34, 590-597. doi:10.1016/ j.medengphy.2011.09.005.

Williams, J. W., Plassman, B. L., Burke, J., Holsinger, T., \& Benjamin, S. (2010). Preventing Alzheimer's disease and cognitive decline. Evidence Report/Technology Assessment. Rockville (MD): Agency for Healthcare Research and Quality (US)

Wilson, R. S., Scherr, P. A., Schneider, J. A., Tang, Y., \& Bennett, D. A. (2007). Relation of cognitive activity to risk of developing Alzheimer disease. Neurology, 69, 1911-1920.

Zammit, S., Lewis, G., Dalman, C., \& Allebeck, P. (2010). Examining interactions between risk factors for psychosis. The British Journal of Psychiatry, 197, 207-211. 197/3/207. 